\title{
Wissenschaftstheoretische Aspekte einer Definition von Geographie
}

Am 22.12.1980 hat das Geographische Institut der Universität Zürich durch Konsens in einem institutionellen Akt folgende Definition beschlossen:

Die Geographie untersucht Natur und Gesellschaft mit dem Ziel, räumliche Systeme und Prozesse zu erklären.

Diese Definition war für mich der Anlass, um über disziplingeschichtliche und erkenntnistheoretische Hintergründe einer Definition zu sprechen.

\section{Forschungslogische versus institutionelle Aspekte}

Die Definition einer Fachwissenschaft beinhaltet die stillschweigende Voraussetzung, dass sich Wissenschaften voneinander abgrenzen lassen. Der Einteilung von Wissenschaft in einzelne Fachwissenschaften steht jedoch entgegen, dass seit 1945 in verschiedenen Fachwissenschaften, so auch in der Geographie, interdisziplinäre Strömungen aufgetreten sind. "Systematisch erzeugt man Interdisziplinarität durch adjektivische Kopplung wie "Politische Psychologie", ... "Mathematische Linguistik" ... (Lorenzen 1974/133). Dieser Trend ist teilweise so weit vorangeschritten, dass die Abgrenzung einzelner Fachwissenschaften aus forschungslogischen Gründen in Frage gestellt werden kann. Damit ist aber die Notwendigkeit der Definition von Fachwissenschaften nicht verschwunden, da die Wissenschaft institutionell in Abteilungen unterteilt wird. Bei einer solchen Definition wirken institutionelle und forschungslogische Komponenten mit. Damit ist sowohl der wissenschaftstheoretische als auch der wissenschaftssoziologische Aspekt der Entwicklung mit in Betracht gezogen. "Aber die Institution ist natürlich immer ... viel langsamer als die Repräsentanten ihres forschungslogischen Gewissens..." (Eisel 1981/188). Aus institutioneller Sicht hat die Definition eines Fachgebietes die Funktion, verschiedene Abteilungen voneinander abzugrenzen. Oft wird dabei so verfahren, dass aus der Totalität der Welt ein Objektbereich ausgegliedert wird, der dann als spezifisches Forschungsobjekt einer Fachwissenschaft bezeichnet wird.
"In den meisten Diskussionen über die Natur der Geographie und ihr Untersuchungsgebiet sind zwei Themen dominant, ein Interesse an 'Raum' und ein Interesse an der Beziehung zwischen Mensch und Natur" (Sayer 1979/20, Uebersetzung vom Verfasser). Disziplingeschichtlich hat sich das Schwergewicht von der Mensch-Natur Beziehung auf den Raumbezug verlagert (Sayer 1979/20). Erdkunde entstand "als eine theoretische Spekulation über das Mensch-Natur Verhältnis" (Eisel 1981/177). Damit ist es eine Frage der Gewichtung, ob die Geographie den Natur- oder den Geisteswissenschaften zugeordnet wird. An der Universität Zürich ist das Geographische Institut den Naturwissenschaften zugeordnet. Disziplingeschichtlich ist dies durchaus begründbar. Heute ist diese Zuordnung nicht mehr unbedingt selbstverständlich, da sich der sozialwissenschaftliche Zweig der Geographie stark entwikkelt hat. An einigen anderen Universitäten der Schweiz ist die Geographie den Geistes- und Sozialwissenschaften zugeordnet.

"Die Grundannahmen des Faches der Geographie insgesamt, die sich aus ihrem 'System' und trennscharfen Grenzen zu anderen Disziplinen legitimierten, wurden in Frage gestellt durch den Beginn der fachinternen Diskussion ... und durch den drohenden externen 'Positionsverlust' im Fächerkanon der Schule, Hochschule und angewandten Wissenschaft" (Rhode-Jüchtern 1975/10). Disziplingeschichtlich ist seit den 50er Jahren eine immer stärkere Aufspaltung in die Teildisziplinen physische- und Sozialgeographie zu verzeichnen. Dies hat soweit geführt, dass von verschiedenen Vertretern die Spaltung des Faches gefordert wurde. Die Krise der Geographie, der Positionsverlust und die Spaltungstendenzen haben zu einer Legitimationsangst geführt, die schon seit längerem latent vorhanden war und sich vor allem institutionell ausgewirkt hat. Um sich als Institution durchsetzen oder mindestens behaupten zu können, versuchten die Geographen ein Forschungsobjekt vorzuweisen, welches in der Wirklichkeit vorgegeben ist (cf Hard 1973/11).

Herbert Wanner, Geographisches Institut der Universität Zürich, Postfach, 8033 Zürich. 
Diese Aufspaltungstendenz ist auch für die Forschergemeinschaft des Geographischen Instituts kennzeichnend. Extreme wie Pollen- und Cl4-Analyse im Rahmen der Postglazialforschung, digitale Satellitenbildverarbeitung, Tourismus in Entwicklungsländern und Erfolgskontrollen von regionalen Planungsstrategien sind am selben Institut vertreten. Dieses setzt sich also aus Forschergruppen mit verschiedensten fachlichen Interessen zusarmen. In der Definition des Fachgebietes mussten dann genannte divergierende Interessen auf einen Nenner gebracht werden. Das Problem wurde so gelöst, dass der Objektbereich so weit gefasst wurde, bis von keiner Forschergruppe mehr Einwände kamen. So wurde sichergestellt, dass jeder mit seiner gegenwärtigen Forschungstätigkeit auch unter der neuen Definition weiterarbeiten konnte wie bis anhin. Die Definition sollte den Grundkonsens aller Beteiligten zum Ausdruck bringen. Man ging dabei nicht nach einem protokollarisch festgelegten Verfahren vor, dh. weder nach methodischen noch nach systematischen Gesichtspunkten. Damit ist in dieser Definition nicht die forschungslogische, sondern die institutionelle Komponente stark betont. Dabei hätte gerade die disziplingeschichtliche Arbeit Anregungen für die eigene Forschung bringen können. In der vorliegenden Form hat die Definition vorwiegend eine legitimatorische Funktion gegen aussen, gegen innen bringt sie den Machtkampf zwischen verschiedenen Forschergruppen zum Ausdruck. Eine neue Definition soll ja nicht nur die Funktion haben, abzugrenzen, sondern vage ausgedrückt, erste Hinweise über Problemstellungen, Grundbegriffe und Methoden einer Wissenschaft zu geben.

Betrachtet man die forschungslogische Komponente, so stellt man fest, dass "die Aufteilung der Welt oder der Erscheinungen und die Einteilung der Wissenschaften selbst immer weniger anthropomorph werden." "Die Entanthropomorphisierung beruht aber nicht allein auf der Benützung hochempfindlicher Geräte und der Ausdehnung des Variablenbereichs. Die Wissenschaften stellen Hypothesen und Theorien über die Welt auf, die zwar unsere Erfahrungen erklären sollen, die sich aber in mehrfacher Hinsicht von den Hypothesen der Alltagserfahrung unterscheiden. Auch hier versuchen wir, über das sinnlich Wahrnehmbare hinauszugehen" (Vollmer 1980/168). Der Uebergang von der physiognomisch orientierten Landschaftsgeographie zu den modermen Ansätzen der Geographie, also der Paradigmenwechsel, kann im Lichte dieser Entanthropomorphisierung betrachtet werden.

Neben den institutionellen und den forschungslogischen Einflüssen auf eine Definition, wollen wir uns nun einem wissenschaftsexternen Einfluss zuwenden. Nach Schultz (cf 1980/272) ist die Abhängigkeit der Definition vom Zeitgeist in der Geographie allgemein anerkannt. Die wenigsten sähen jedoch darin einen Grund, den Gedanken von der ewigen Geographie und ihrem natürlich vorgezeichneten Entwicklungsgang aufzu- geben. Die einheitliche Geographie kann es jedoch nicht geben; 'Geographie' ist ein historischer Begriff mit bestimmten Inhalten, welcher von Person und Ort abhängig ist. Die Definition von Geographie ist nicht ausschliesslich Angelegenheit von Geographen, sondern die allgemeine gesellschaftliche Situation legt der Geographie Fragen vor. "Das Fach Geographie überlebt nicht durch blosse Objektdefinition. An die Stelle traditionell fixierter Forschungsgegenstände müssen problemorientierte Fragestellungen treten. Derartige Fragestellungen machen Fachgrenzen überflüssig" (Geografiker 1969 Heft 3, S. 15 zitiert nach Schultz 1980/263).

\section{Von der Einheit zur pluralistischen Heterogenität}

Die Gliederung der Geographie in ihre Teilgebiete war früher eine vieldiskutierte Frage. In der Gegenwart ist sie abgelöst worden durch die Kontroverse um die Heterogenität des Faches. Nach Johnston (1979/188) hat die Humangeographie gegenwärtig nicht eine einzelne disziplinäre Matrix. Sie besass dies auch seit dem Zweiten Weltkrieg nicht. Es gibt aber mehrere disziplinäre Matrizen, die um eine stabile Position oder um Dominanz konkurrierten. "Mit der Behavioral Geography, der Welfare Geography, der Radical Geography und anderen 'Geographies' ist ein ganzer Strauss von Ansätzen im Fach aufgetaucht" (Beck 1981/165). Erste Indizien dieses Auffächerungstrends versuchten einzelne Geographen mit dem Venn-Diagram zu veranschaulichen (z.B. Bartels 1968/181). Für Bartels $(1980 / 34)$ ist die zeitgenössische Geographie gekennzeichnet durch eine Heterogenität und Inkommensurabilität. " "Es bleibt... nur die pluralistische Sicht auf eine ... logisch nicht zu ordnende Fülle älterer und neuerer Forschungsansätze... (Bartels 1980/ 44). Die Auffassung von der Einheit der Geographie deckt sich mit der positivistischen Annahme der 'unity of method' (Sayer 1979/20). Im Uebergang von der Geographie als Einheit zur heterogenen Geographie zeigt sich auch eine gewisse Abkehr vom Positivismus, indem mit einer Vielzahl von Ansätzen auch eine methodische Pluralität erreicht wurde. Diese Entwicklung von der Einheit zur pluralistischen Heterogenität kann nun nicht mehr befriedigend mit dem Paradigmenkonzept von Kuhn erklärt werden. So verwirft z.B. Johnston das Paradigmenkonzept (1979/ 187). Für Feyerabend bleibt nach einer Reihe von wissenschaftsgeschichtlichen Studien nur noch die vieldiskutierte Feststellung übrig: 'anything goes' (1980/100 ff).

Trotz dieser Heterogenität in den modernen Ansätzen der Geographie versuchte das Institut eine Definition zu finden, welche die Interessen der verschiedenen Forschergruppen 'unter einem Hut' vereint. Dies kann als Versuch gewertet werden, die fachliche Einheit aus der Aera der Landschafts- und Länderkunde in die heu- 
tige Zeit zu retten. Mit den Begriffen 'System', 'Prozess' und 'erklären', welche eine methodologische Einheit repräsentieren, kann die Definition nicht für einen spezifischen modernen Ansatz stehen. Die Heterogenität der Geographie wird mit der Definition eben gerade nicht angesprochen, sondern ihr Ausgangspunkt ist die Einheit des Faches. Bartels zeigt, dass sich die Landschaft in einer systemtheoretischen Auffassung aufgelöst hat (1968/70) Auch Schultz und Brunner sprechen von einer Selbstauflösung der Landschaftsgeographie. Dadurch, dass die Begriffe 'Landschaft' und 'Untersuchungsgebiet' austauschbar geworden sind, ist 'Landschaft' zur Leerformel degeneriert (cf Schultz 1980/251 ff, Brunner 1981/147 ff). Ein Begriff, welcher nichts einschränkt, ist auch kein operationales Instrument mehr, er hat seinen Inhalt verloren. Wenn die Vorgabe eines Untersuchungsgebietes die einzige Einschränkung ist, dann kann in der Geographie jedes beliebige Problem untersucht werden, es gilt "Geography is what geographers do". Damit hat die Geographie auch die Problemstellung verloren. "Die Landschaftsgeographen haben... gar keine Probleme mehr entdeckt (Beck 1981/166), dh. es fanden keine Problemverschiebungen mehr statt, welche einen Fortschritt hätten ausmachen können. Lakatos nennt "eine Problentverschiebung progressiv, wenn sie sowohl theoretisch als auch empirisch progressiv ist, und degenerativ, wenn das nicht der Fall ist" (Lakatos 1974/155 ff).

In der Definition sind an die Stelle des Landschaftsbegriffes die Begriffe 'Natur' und 'Gesellschaft' getreten, womit nicht nur die Spaltung des Faches, sondern auch das Ende der Landschaftsgeographie bekundet wird. Hinter dieser scheinbaren Differenz zwischen 'Landschaft' sowie 'Natur und Gesellschaft' steht verbindend, dass beide für eine nicht genau fassbare Totalität stehen. Die Definition sagt jedoch nichts aus über das Verhältnis zwischen Mensch und Natur. Damit bleibt eine zentrale Problemstellung mit alter Tradition unberücksichtigt.

\section{Erkenntnistheoretischer Zugang zur Definition}

Erkenntnistheorie befasst sich mit der Frage, wie eine Wissenschaft zu den Grundbegriffen und zu ihren Objekten, dh. letztlich zu Erkenntnis komt. "...Architektur, Psychologie, Soziologie, Politologie etc. (sind) mögliche Partner der Geographie im interdisziplinären Zusammenhang.. .., Erkenntnistheorie, Wissenschaftstheorie und Methodologie dagegen (sind) überdisziplinär.... Die Theorie und Methoden der Sozialwissenschaft gehen damit etwa die Geographie in jedem Falle etwas an, unabhängig von der jeweiligen Grenzbestimmung zur Soziologie" (Rhode-Jüchter 1975/ 18). Das Objekt einer Wissenschaft wird nicht einfach vorgefunden, sondern es ist etwas Theoretisches und muss vom Forscher konstruiert werden, dh. eine Wissenschaft erfordert als erkenntnistheoretischen Akt die Konstitution eines Gegenstandsbereiches. Gegenstände müssen be- grifflich gefasst werden. Sie sind sprachliche und damit auch theoretische Konstrukte. "Wissenschaften operieren meistens mit einem Objekt, das von ihnen so dargestellt wird wie ein Realobjekt. Normalerweise folgen aus diesem Realobjekt auch die sogenannten adäquaten Methoden und die Fragestellungen. Die klassische Landschaftskunde ist ein sehr gutes Beispiel dafür... " (Eisel 1981/185). Nach Bartels ist auf dem Hintergrund eines realistischen statt nominalistischen Verständnisses die Theoriebildung in der Geographie vernachlässigt worden (cf 1980/35). Der realistischen Auffassung steht die nominalistische gegenüber, bei welcher "der 'Name' des Objektes im Sinne eines definierten Objektes aufgefasst" wird (Eisel 1981/ 185). Oder anders gesagt gilt nach nominalistischer Auffassung, dass wir erst mit den Begriffen die Welt in Gegenstände aufteilen. Anschliessend an die Konstitutionsphase des Gegenstandes folgt der Aufbau einer adäquaten Methodologie. oft wird jedoch bereits der Gegenstand aufgrund gewisser methodologischer Ideale gefunden.

Hard beschreibt die stillschweigenden Voraussetzungen, die bei der Frage 'Was ist Geographie' gemacht werden (1973/9-32). Eine dieser Voraussetzungen ist, dass die Geographie einen in der Wirklichkeit vorgegebenen Gegenstand hat, und dass dieser Gegenstand disziplinhistorisch dauerhaft ist. So gesehen ist die Auffassung von Geographie und damit von Wissenschaft eine realistische. Hard zeigt, dass ein Zusammenhang zwischen wissenschaftlichen Fragen und den Gegenständen der Wissenschaft besteht. Daraus ergeben sich Frage-Antwort Komplexe. Aendern sich die Frageweisen einer Wissenschaft, so ändern sich auch die Gegenstände. Frageweisen sind bestimmt durch das Erkenntnisinteresse des Forschers. Die Frage nach dem Forschungsobjekt der Geographie wurde oft als ontologisches Problem aufgefasst. Es wurde versucht, das Forschungsobjekt als ein Abbild der Wirklichkeit auszuweisen. Solche ontologische Aspekte äussern sich etwa in den Fragen nach dem 'Wesen der Landschaft', dem 'Gegenüber von Mensch und Welt als dem Ganzen alles Seienden'. Diese Ausrichtung kann im Zusammenhang mit der Legitimations- und Positionskrise der Geographie betrachtet werden. Diese Krise wirkt sich, wie oben gezeigt wurde, vor allem auf institutionelle Belange aus. Dies hat dazu geführt, dass beim Versuch, den "Forschungsgegenstand durch die Wirklichkeit verbindlich vorzugeben, (dieser) ontologisch überhöht wurde" (Hard 1973/11 ff). Von der ontologischen Ueberhöhung kann eine Linie zur Totalität der Landschaft und damit zur Selbstauflösung gezeichnet werden.

Nach Hard hat dieser Frage-Antwort Komplex typische Antwortformen. Er untergliedert die Antworten in die Teile A und B, wobei A die Methodologie, die Forschungslogik, den 'Context of Justification', also das 'Wie' der Geographie meint. Teil B stellt den Gegenstand, das 'Universe of Discourse', die Problemstellung und die Perspektive dar, also das 'Was', dh. es ist eine Wert- 
entscheidung darüber, was behandelt werden soll. Wenn wir nun die Definition des Geographischen Instituts anhand dieses Schemas von Hard besprechen, so sind die Begriffe 'System', 'Prozess', 'erklären' und 'räumlich (als Ordnungsschema von Daten) 'dem Teil A, die Begriffe 'Natur' und 'Gesellschaft' dem Teil B zuzuordnen. Wenden wir uns nun dem Teil A zu. Hard sagt von den Begriffen 'Natur' und 'Gesellschaft', dass sie Gegenstände darstellen, aus denen die vorwissenschaftliche welt aufgebaut ist. Damit sind die Gegenstände noch nicht als theoretische Konstruk te formuliert. Wenn wir nun zu Teil B wechseln, so erkennen wir in den Begriffen 'erklären', 'System', 'Prozess' und 'räumlich' die methodologische Grundlage des Faches.

\section{Zu den einzelnen Begriffen der Definition}

Nach Bartels (1980/37) ist der Konsistenzgrad und die definitorische Durchgestaltung des Begriffsmusters einer Forschungsrichtung eine wichtige Kategorie ihrer Beurteilung. "Die Bestimmung eines Begriffs kann nicht wahr oder falsch sein, wohl aber mehr oder weniger sinnvoll. Sinnvoll ist es für ein Ziel, das mit der Verwendung des Begriffs verfolgt wird. Kann man die Ziele der begrifflichen Arbeit angeben, so hat man folglich eine Möglichkeit, für oder gegen eine Begriffsbestimung $\mathrm{zu}$ argumentieren. Diese Ziele können ausserwissenschaftliche oder innnenwissenschaftliche sein" (Schwenmer 1981/ 87). Ausser dem theoretischen Ziel 'zu erklären' enthält die Definition kein praktisches Ziel und damit auch keine Problemstellung mit lebensweltlichen Problemen wie z.B. Siedlungs-, Bevölkerungs-, Umwelt- oder Planungsproblemen. "...ein komplexes Problem wie z.B. "die Verstädterung" oder "die Umweltkrise" ist als Integral aller Forschungsrichtungen... weiter entwickelt als die Ergebnisse einzelner Disziplinen oder Fragestellungen" (Rhode-Jüchtern 1975/29). Deshalb können solche Probleme auch nicht die Probleme einer einzigen Wissenschaft sein, dh. die Problemstellung entbindet nicht davon, den spezifischen Aspekt, dh. das Interesse an diesem Problem zu formulieren. Es kann nicht genügen, die Grundbegriffe aus der Alltagssprache zu entlehnen. Wir verwenden "zur Formulierung unserer Theorien eine eigens dafür konstruierte wissenschaftliche Sprache. Wir wissen ..., dass unsere natürliche Sprache.... für die genaue und kritische Erfassung der Welt nicht ausreicht. Manche Begriffe, die bereits in der Umgangssprache eine vage Bedeutung haben, ... , werden präzisiert. Andere ... wie Entropie... werden völlig neu geschaffen, weil die Alltagssprache gar kein wort für die betreffende... Struktur bereitstellt" (Vollmer 1980/168).

Es sollen hier noch die einzelnen Begriffe der Definition diskutiert werden. Bemerkenswert ist, dass der Begriff 'Beschreibung', welcher die Definitionen von Geographie so lange dominiert hatte, nicht vorkomt. An seine Stelle ist der Begriff 'erklären' getreten, womit eine Orientierung auf Theorie bekundet wird.

Mit dem Begriff 'räumlich' ist das spezifisch Geographische in der Definition verankert. "Ich habe sofort den Daumen auf das wort 'räumlich' gehalten und habe gefragt, was passieren würde, wenn man dies wegliesse. Es wäre eine Definition gewesen, in die man ganz viele Wissenschaften hätte einsetzen können... Was mich an der Definition stört, ist, dass der Begriff 'räumlich' zu wenig seine Richtung zeigt und $z \mathrm{u}$ formal ist." (Eisel 1981/189). "Meine bisherige Strategie war... zu zeigen, was jeder Begriff von Raum impliziert, was er gesellschaftstheoretisch heisst und was daraus folgt..." (Eisel/ 188). Wenn jedoch 'Raum' nur die Funktion hat, die Geographie als eigenständige Wissenschaft zu legitimieren, so kann dieser 'Raum' auch als Freiraum verstanden werden, um konkrete Probleme und Interessen zu verfolgen oder auch um Träume und Utopien bearbeiten zu können. Als paradigmatisches Beispiel kann 'Utopia' von Thoma Morus angeführt werden, in welchem eine stark geographisch ausgelegte gesellschaftliche Utopie vorgelegt wurde. Damit der Raumbegriff jedoch nicht undurchsichtig und so zum 'trojanischen Pferd' wird, muss Transparenz gefordert werden. Dies kann durch verstärkte wissenschaftliche Diskussionen um die Inhalte und Positionen der Geographie erreicht werden.

'System' kann definiert werden als eine Menge von Elementen, die in Relation stehen. Die Elemente sind mit bestimmten Eigenschaften versehen. Systemtheorie ist eine formale Sprache, die aus sehr wenigen Begriffen besteht. Ihr Vorteil besteht in der Operationalisierbarkeit. Ihr Nachteil ist, dass jedes Problem (u.a. auch eine Fülle von Individuellem) reduziert werden muss auf die Grundbegriffe 'Element', 'Eigenschaft' und 'Relation'.

Nach Baker (1981/439) ist der Raumbegriff ebensowenig Grundlage der Geographie wie der Zeitbegriff Grundlage der Geschichte ist. Mit dem Begriff 'Prozess' wird die Dimension 'Zeit' abgedeckt. Der Einschluss der Zeitdimension ergab sich in verschiedenen historischen Schulen der Disziplin, in denen die Genese, dh. die geschichtlich-idiographische Kausalerklärung der Gegenwart aus der Vergangenheit im Vordergrund stand (Bartels 1968/90). Die Zeitdimension kann auf verschiedenen Arten aufgefasst und in einen geographischen Ansatz eingebaut werden. Bird (1980/137) nennt zwei grundsätzlich verschiedene Typen von Prozessen. Erstens, den mechanistischen, welcher durch eine frühere Ursache und eine spätere Wirkung gekennzeichnet ist. Zweitens den teleologischen Prozess, welcher an einem Zielzustand orientiert ist. Die Definition lässt offen, welcher der beiden Prozesstypen gemeint ist. 
Nach Hard stellen die Begriffe Natur und Gesellschaft die Gesamtheit der Realität dar. Wenden wir uns nun dem Begriff 'Natur' zu. "Die Naturwissenschaften konstituieren... ihren Gegenstand selbst, dh. sie bestimmen die ... Grundbegriffe ...". Darnit "erzeugen... die Naturwissenschaften erst ihre 'Natur', die nicht die Natur vor unseren Augen ist, sondern die Natur, wie sie sich uns erst durch dieses unser unterscheidendes und herstellendes Handeln zeigt" (Schwemmer 1981/89). So kann "Natur im Sinne der neuzeitlichen Naturwissenschaft betrachtet (werden), dh. unter Abstraktion von der konkreten Phänomenologie als abstraktes System von Kraftausübung, Energiefluss usw." (Eisel 1981/176). Oder auch Natur als "System von Bewegung und Kraftausübung" (Eisel 1981/180).

Für den Begriff 'Gesellschaft', welcher in mehreren Disziplinen verwendet wird, gelten ähnliche semiotische (sprachsymbolische) Probleme wie für den Landschaftsbegriff (Bartels 1980/ 37). Als mehrdeutiger Grundbegriff der Soziologie sind etwa die miteinander verflochtenen Sozialbeziehungen zwischen Menschen gemeint. Ge- sellschaft kann in einem Wortfeld angesiedelt werden zusammen mit Begriffen wie Gruppe, Klasse, Institution, Staat und Gemeinschaft. In verschiedenen Gesellschaftstheorien stehen sich aber verschiedene wissenschaftstheoretische und politische Positionen gegenüber.

Im Folgenden kann versucht werden, die in der Definition vorgeschlagenen Grundbegriffe zusammen mit konkreten Problemstellungen zu theoretischen Ansätzen zu verknüpfen. Die Grundbegriffe können zueinander in Beziehung gesetzt und zum Aufbau eines Begriffsapparates verwendet werden. Beispielsweise kann nach dem Ansatz von Eisel versucht werden, Zusammenhänge zwischen Raumkonzeptionen und Gesellschaftstheorien aufzudecken. Oder man versucht in einem Forschungsprogramm Zusammenhänge zwischen Gesellschaftstheorien und Naturbegriffen zu finden. Damit könnte man nicht nur das in der jüngeren Vergangenheit etwas vernachlässigte Verhältnis zwischen Mensch und Natur wieder aktualisieren, sondern auch mögliche Erklärungen für Umweltprobleme anbieten.

\section{Zitierte Literatur}

BAKER, A. (1981): An historico-geographical perspective on time and space and period and place. In: Progress in Human Geography, no 3, 439-443.

BARTEIS, D. (1968): Zur wissenschaftstheoretischen Grundlegung einer Geographie des Menschen. Wiesbaden.

BARTEIS, D. (1980) : Geographie - Die Fachwissenschaft als Bezugswissenschaft seiner Fachdidaktik. In: G. Kreuzer: Didaktik des Geographieunterrichts. Hannover.

BECK, G. (1981) : Zur Theorie der Verhaltensgeographie. In: Geographica Helvetica Nr. 4, 155166.

BIRD, J. (1981): The target of space and the arrow of time. In: Transactions, Inst. Br. Geogr. N.S.6, 129-15l.

BRUNNER, H.R. (1981): Die Zürcher Landschaftsschule. In: Geographica Helvetica Nr. 4, 146154 .

EISEL, U. (1981): Zum Paradigmenwechsel in der Geographie. In: Geographica Helvetica Nr. 4, 176-190.

FEYERABEND, P. (1980): Erkenntnis für freie Menschen. Frankfurt.

HARD, G. (1973): Die Geographie, Eine wissenschaftstheoretische Einführung. Berlin.
JOHNSTON, R.J. (1979) : Geography and Geographers, Anglo-American Human Geography since 1945. London.

LAKATOS, I. (1974): Falsifikationismus und Methodologie der Forschungsprogramme. In: LAKATOS, I., MUSGRAVE, A. (1974): Kritik und Erkenntnisfortschritt. Braunschweig.

LORENZEN, P. (1974): Konstruktive Wissenschaftstheorie. Frankfurt.

RHODE-JUECHTERN, T. (1975) : Geographie und Planung. In: Marburger Geographische Arbeiten, Heft 65 .

SAYER, A. (1979): Epistemology and Conceptions of People and Nature in Geography. In: Geoform, Vol. 10, no 1 .

SCHULTZ, H.D. (1980): Die deutschsprachige Geographie von 1800 bis 1970, Abhandlungen des Geographischen Institutes - Anthropogeographie, FU Berlin, Bd 29.

SCHWEMMER, O. (1981): Vernunft, Handlung und Erfahrung. Minchen.

VOLIMER, G. (1980) : Evolutionäre Erkenntnistheorie. Stuttgart.

WEINGARTEN, E., SACK, F. und SCHENKEIN, J. (1976): Ethnomethodologie, Beiträge zu einer Soziologie des Alltagshandelns. Frankfurt. 